\title{
Inducing topological order in a honeycomb lattice
}

\author{
T. Pereg-Barnea ${ }^{1}$ and G. Refael ${ }^{2}$ \\ ${ }^{1}$ Department of Physics, McGill University, Montreal, QC, Canada \\ ${ }^{2}$ Department of Physics, California Institute of Technology, 1200 E. California Boulevard, MC114-36, Pasadena, California 91125 , USA
}

(Received 23 November 2010; revised manuscript received 7 February 2012; published 22 February 2012)

\begin{abstract}
We explore the possibility of inducing a topological insulator phase in a honeycomb lattice lacking spin-orbit interaction using a metallic (or Fermi gas) environment. The lattice and the metallic environment interact through a density-density interaction without particle tunneling, and integrating out the metallic environment produces a honeycomb sheet with in-plane oscillating long-ranged interactions. We find the ground state of the interacting system in a variational mean-field method and show that the Fermi wave vector $k_{F}$ of the metal determines which phase occurs in the honeycomb lattice sheet. This is analogous to the Ruderman-KittelKasuya-Yosida (RKKY) mechanism in which the metal's $k_{F}$ determines the interaction profile as a function of the distance. Tuning $k_{F}$ and the interaction strength may lead to a variety of ordered phases, including a topological insulator and anomalous quantum-Hall states with complex next-nearest-neighbor hopping, as in the Haldane and the Kane-Mele model. We estimate the required range of parameters needed for the topological state and find that the Fermi vector of the metallic gate should be of the order of $3 \pi / 8 a$ (with $a$ being the graphene lattice constant). The net coupling between the layers, which includes screening in the metal, should be of the order of the honeycomb lattice bandwidth. This configuration should be most easily realized in a cold-atoms setting with two interacting Fermionic species.
\end{abstract}

DOI: 10.1103/PhysRevB.85.075127

PACS number(s): 71.10.Fd, 71.10.Hf, 71.10.Pm

\section{INTRODUCTION}

In 2005 Kane and Mele ${ }^{1,2}$ proposed that graphene, a one-atom-thick graphite, can exhibit helical edge states that are protected against weak perturbations by topology. This idea stems from the Haldane model ${ }^{3}$ of a quantum Hall state without magnetic field. This special topological phase would have emerged as the ground state of graphene if the intrinsic spin-orbit coupling was large enough and in particular, larger than the Rashba spin-orbit coupling. Unfortunately, ab initio calculations $^{4}$ have found that this requirement is far from being fulfilled in graphene and the desired intrinsic spinorbit coupling is smaller than $0.001 \mathrm{meV}$ (about $0.01 \mathrm{~K}$ ). Nevertheless, this direction has led the way to a wealth of theoretical predictions ${ }^{5-9}$ of topological states. In parallel, experiments have shown that robust helical edge states exist in three dimensions ${ }^{10,11}$ and in two-dimensional quantum wells. ${ }^{12,13}$

Despite the fact that graphene's intrinsic spin-orbit coupling may be too weak to produce the desired topological insulator phase, the hope to achieve such a phase in the honeycomb lattice has not died. In this work we explore the possibility of engineering a honeycomb-lattice-based or graphene-based topological insulator by employing an appropriate environment. Our results are relevant either to graphene with a metallic gate, or to a Fermi gas residing in a honeycomb optical lattice (see, e.g., Ref. 14), and interacting with a second Fermi gas of similar (two-dimensional) density that does not couple to the optical lattice. Note that another path for producing topological states in optical lattices was explored in Ref. 15.

An important ingredient for topological behavior is a bulk gap that arises through avoided level crossing between two bands and a resulting band inversion. A half filled honeycomb lattice (e.g., undoped graphene) has a band crossing at the two Dirac points ${ }^{14,16}$ but without any band gap. An opening of a gap in the bulk is possible in a few ways, for example by substrate effects ${ }^{17}$ or a large lattice distortion. ${ }^{18}$ However, not all gaps are alike. In a two valley model like graphene, nontrivial topology can only occur if the gap function changes sign between the two valleys. This sign change can occur, for instance, due to intrinsic spin-orbit coupling. On the lattice, this coupling may be approximate by imaginary hopping between next-nearestneighbor sites and this yields the Kane-Mele model. The sign of the imaginary hopping depends not only on the direction of the hopping but also on whether the path from one site to another contains a left or right turn. In momentum space, the imaginary hopping takes the form of a function that changes sign between Dirac valleys, and between spin polarizations.

Apart from intrinsic spin-orbit coupling, this type of directional hopping term has been found to arise from an interaction between next-nearest-neighbor sites. ${ }^{19,20}$ It has also been found that interactions may lead to a topological insulator in a decorated honeycomb and kagome lattices. ${ }^{21}$

The main obstacle for realizing an interaction-driven topological insulator is the required interaction profile in real space. In a honeycomb lattice, for example, this amounts to an interaction that is strongest on next-nearest-neighbor bonds. When this requirement is not fulfilled, other phases (density waves, lattice distortions, etc.) may occur. An on-site repulsion $U$ (arising due to Coulomb interactions in electrons, or on-site scattering for cold atom), enhances the tendency for magnetic ordering. The next strongest interaction (for electrons) is the nearest-neighbor repulsion, $V_{1}$, which tends to favor charge/spin density waves. A second-nearest-neighbor interaction $V_{2}$, however, enhances precisely the propensity to induce an imaginary hopping term leading to the topological phase. It is hard to find, however, lattice systems where the second-nearest-neighbor interaction trumps the shorter term repulsions; therefore the topological phase is not likely to arise due to intrinsic interactions. In addition, if $V_{1}$ and $V_{2}$ are of similar order, a Kekulé bond order may occur. ${ }^{20}$ Extensive studies 
on interacting graphene have shown a variety of competing phases. $^{22-24}$ Below we discuss the effects of producing the longer range interactions required for topological order using the Friedel oscillations and Ruderman-Kittel-Kasuya-Yosida (RKKY) interactions occurring in a metallic (Fermi-gas) environment. Our analysis considers the possibility of forming both topological phases as well as other competing phases.

\section{TUNING THE INTERACTION}

In this work we propose a setup in which a "knob" can be turned to control the relative ratio between the different interaction terms. Our idea is motivated by the RKKY ${ }^{25-27}$ interaction in metals. The original RKKY model was written to describe how impurity spins interact with each other over a long distance through a medium of electronic states. The interaction does not simply fall as a power law of the distance between the spins like the usual Coulomb interaction but instead oscillates. The oscillations can be viewed as Friedel oscillations in the metal caused by the impurity spins. The interaction profile depends on the polarization of the metal and its functional form is $-\cos \left(2 k_{F} r\right) / r^{3}$ in three dimensions and $-\cos \left(2 k_{F} r\right) / r^{2}$ in two. The Fermi gas background is considered as a simple parabolic band.

With this scenario in mind we propose to generate interactions in the honeycomb lattice that are mediated by an additional Fermi gas or metallic gate. An electronic realization of such a system consists of a metallic layer, which is put above a honeycomb lattice such that the quasiparticles in it interact with the metallic-gate quasiparticles without direct hopping between the two systems. A more promising avenue, which our analysis more readily addresses, is a cold-atoms realization, consisting of two different species of fermions, only one of which is trapped in an optical honeycomb lattice, with a contact interaction between the two species. The effect of the coupling between the honeycomb fermions and the free gas can be found by integrating out the metallic degrees of freedom. This results in the honeycomb fermions acquiring a long-range interaction whose magnitude depends on the polarization of the free Fermi gas. For simplicity, we will assume that the fermions are electron-like, and refer to them as electrons and fermions interchangeably below.

\section{FORMALISM}

Our starting point is electrons hopping on a honeycomb lattice and interacting through an on-site density-density interaction with fermions in a metallic layer:

$$
\mathcal{H}=-t \sum_{\langle i j\rangle} \sum_{\sigma} c_{i \sigma}^{\dagger} c_{j \sigma}+\sum_{\mathbf{k} \sigma} \epsilon_{\mathbf{k}} d_{\mathbf{k} \sigma}^{\dagger} d_{\mathbf{k} \sigma}+\alpha \sum_{i} n_{i}^{c} n_{i}^{d},
$$

where $c_{i \sigma}^{\dagger}$ creates an electron with spin $\sigma$ on the honeycomb lattice site $i, d_{\mathbf{k} \sigma}^{\dagger}$ creates an electron with spin $\sigma$ in a Bloch state $\mathbf{k}$ of the metal and $n_{i}^{c}$ and $n_{i}^{d}$ are the number operators at the position $i$ of the honeycomb lattice and metal electrons, respectively. In the metal this should be understood as a coarsegrained density integrated over a small area $A_{\text {eff }}$ around the position $i$. This area should be estimated by the screening in the metal, or in the cold-atom case, by the probability density of the two species. We set it to the lattice constant $a$ in order to simplify the notation. A larger area may enhance the interaction but will also make the approximate interaction term in Eq. (1) less accurate. We estimate the value of $\alpha^{2}$ in the discussion section and in the Appendix.

In the path-integral language the action is given by a time integration over the Lagrangian, which is derived from the above Hamiltonian and the integration over the metallic Fermion operators $d_{k}$ is possible due to their quadratic form. The result is an interacting theory of quasiparticles on the graphene sheet with their action given by

$$
\int d \tau\left[t \sum_{\langle i j\rangle} \sum_{\sigma} c_{i \sigma}^{\dagger} c_{j \sigma}+\alpha^{2} \int d \tau^{\prime} \sum_{i j} \Pi_{i j}\left(\tau-\tau^{\prime}\right) n_{i}^{c} n_{j}^{c}\right],
$$

where the integration is over the imaginary time variables $\tau$ and $\tau^{\prime}$ and $\Pi_{i j}\left(\tau-\tau^{\prime}\right)$ is the polarization operator of the metal. The interaction above is long ranged and dynamic. Its functional form in momentum and Matsubara frequency space is given by the Matsubara sum of the polarization bubble of the Fermi sea:

$$
\Pi(i \Omega, \mathbf{q})=\int d^{2} k \frac{n_{F}\left(\epsilon_{\mathbf{k}}\right)-n_{F}\left(\epsilon_{\mathbf{k}+\mathbf{q}}\right)}{i \Omega-\epsilon_{\mathbf{k}}+\epsilon_{\mathbf{k}+\mathbf{q}}},
$$

where $n_{F}$ is the Fermi-Dirac occupation number, which includes the chemical potential. This polarization operator leads to a long-ranged, time-dependent interaction in the honeycomb lattice. In the current work, for simplicity, we explore the limit of static interaction. The static limit is valid if the velocity in which information travels in the honeycomb lattice is much slower than the typical velocity in the metal. The comparison can be made in terms of energy. In the graphene sheet we take the energy of the gap that opens as a result of the topological order and in the metal it is the Fermi energy. We found that there is indeed a range of parameters where the developed gap is small compared to the metal's Fermi energy.

\section{METHOD AND RESULTS}

The static limit of the polarization bubble is the famous Lindhard function in two dimensions. ${ }^{28,29}$ It is given by

$$
\Pi(\Omega \rightarrow 0, r)=-\frac{a^{2}}{t} \frac{\cos \left(2 k_{F} r\right)}{r^{2}},
$$

where $r$ is the distance between the interacting sites, $k_{F}$ is the Fermi wave vector of the metal, the $a^{2}$ factor represents the effective interaction area $A_{\text {eff }}$ and $1 / t$ is the estimated metallic density of states at the Fermi level. In order to make a crude estimate of the effective interaction strength in the layer we assume that the interaction between the electrons on the graphene sheet and the metal electrons is $e^{2} / d$ where the distance $d$ is of the order of $10 \AA$. This gives a coupling $\alpha$, which is of the order of the bandwidth $t(2-3 \mathrm{eV})$. However, when screening is taken into account (see the Appendix) the bare coupling is reduced by a factor of $\exp \left(-4 k_{F} d\right)$ and therefore the required distance is much smaller. This poses a great challenge since at small distances tunneling may occur. We present results (Fig. 1) for this order of interactions where $(\alpha / t)^{2}$ is scanned from 0 to 4 .

The standard mean-field decomposition leads to selfconsistency equations that are usually solved by iterations. 


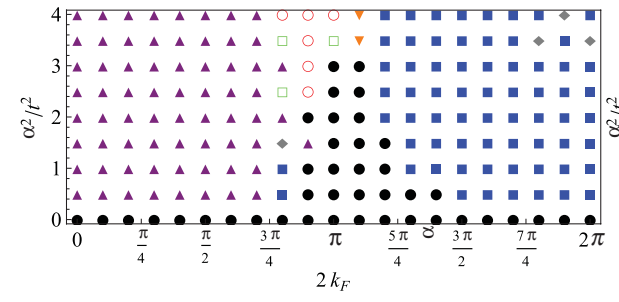

(a) $U=0$

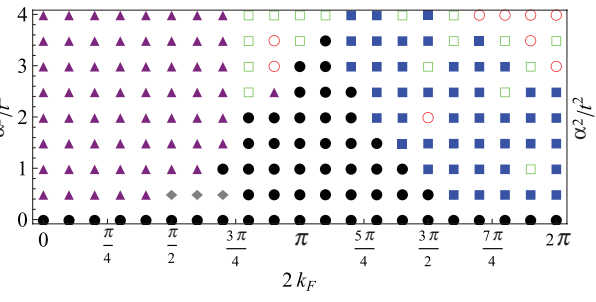

(b) $U=t$

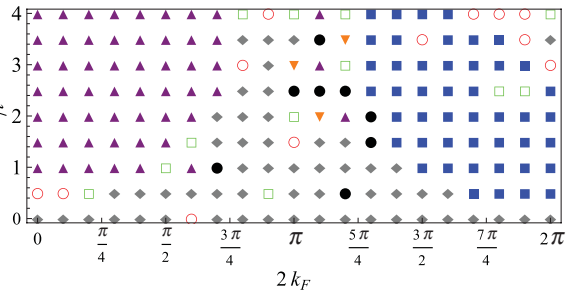

(c) $U=2 t$

FIG. 1. (Color online) Phase diagram for graphene with induced long-ranged interaction of the form of Eq. (4). The $x$ axis is $2 k_{F}$ of the metallic layer (measured in units of $1 / a$ where $a=2.46 \AA$ is the graphene lattice constant), which governs the relative strength of interaction in different distances and the $y$ axis is the overall interaction strength, which is determined by the coupling $\alpha$. The on-site interaction parameter $U$ was scanned. Here we show (a) $U=0$, (b) $U=t$, and (c) $U=2 t$ where $t$ is the hopping amplitude. The different symbols represent different phases with circle (black) semimetal, square (blue) charge-density wave, diamond (gray) spin-density wave, up triangle (purple) superconductor, down triangle (orange) Kekulé, open circle (red) anomalous Hall, open square (green) anomalous spin Hall.

The interaction terms are decomposed in the various channels. For example, the next-nearest-neighbor interaction has been decoupled by Raghu et al. into the desired second-neighbor hopping interaction: $V_{2} c_{i}^{\dagger} c_{i} c_{j}^{\dagger} c_{j} \rightarrow V_{2}\left(\chi_{i j} c_{j}^{\dagger} c_{i}+\right.$ h.c $\left.-\left|\chi_{i j}\right|^{2}\right)$ and the self-consistency equation reads $\chi_{i j}=\left\langle c_{i}^{\dagger} c_{j}\right\rangle$. This decomposition is reasonable, however, one should take care to decouple all interaction terms in all channels. When this is not done correctly the combined effect of multiple terms may result in errors and phases may be missed. Given the drawbacks of the standard mean-field decoupling we adopt the variational mean-field approach. ${ }^{30}$ This is a systematic procedure, which does not suffer from the above problems. We generate a variational wave function that is a solution to a quadratic auxiliary Hamiltonian. This generating Hamiltonian $\mathcal{H}_{\text {gen }}$ contains the graphene nearest-neighbor hopping and a variety of order parameters. These include charge- and spin-density waves in which the two sublattices have different charge/spin density; the Kekule distortion, which is a structural distortion known to occur in carbon nanotubes ${ }^{31}$ and may have interesting topological excitations, ${ }^{32}$ the anomalous Hall or spin Hall state, and superconductivity. Superconductivity is included here since at certain Fermi vectors some interaction coefficients may be attractive. The resulting superconducting state has an interesting momentum-dependent order parameter, which resembles the graphene's band dispersion. Near the valleys the superconducting order parameter has a $p_{x} \pm i p_{y}$ form with the sign changing between the two valleys. ${ }^{33}$ Another interesting suggestion for this region is Kekule superconductivity. ${ }^{34}$

The generating Hamiltonian is a $12 \times 12$ matrix for each momentum $\mathbf{k}$ with the following structure:

$$
\begin{gathered}
\mathcal{H}_{g e n}(\mathbf{k})=\left(\begin{array}{cc}
\hat{h}_{\uparrow} & \hat{g} \\
\hat{g}^{\dagger} & -\hat{h}_{\downarrow}
\end{array}\right), \\
\hat{h}_{\uparrow}=\left(\begin{array}{ll}
H_{A A} & H_{A B} \\
H_{B A} & H_{B B}
\end{array}\right), \quad \hat{g}=\left(\begin{array}{ll}
G_{A A} & G_{A B} \\
G_{B A} & G_{B B}
\end{array}\right),
\end{gathered}
$$

where the $2 \times 2$ structure of $\mathcal{H}_{\text {gen }}$ is the Nambu space with $\hat{h}$ containing the usual particle-hole terms and $\hat{g}$ containing the pairing amplitude. The matrices $\hat{h}$ and $\hat{g}$ are further split into the $A$ and $B$ sublattices and due to the Kekule distortion (which enlarges the unit cell to include three atoms of each sublattice) the matrices $H_{n m}$ and $G_{n m}$ of dimension 3,

$$
\begin{aligned}
H_{A A} & =\left(\begin{array}{ccc}
\rho_{\uparrow} & S_{\mathbf{k} \uparrow} & S_{\mathbf{k} \uparrow}^{*} \\
S_{\mathbf{k} \uparrow}^{*} & \rho_{\uparrow} & S_{\mathbf{k} \uparrow} \\
S_{\mathbf{k} \uparrow} & S_{\mathbf{k} \uparrow}^{*} & \rho_{\uparrow}
\end{array}\right), \\
H_{A B} & =\left(\begin{array}{ccc}
t_{3} & t_{2} e^{i \mathbf{k} \cdot \mathbf{a}_{2}} & t_{1} e^{i \mathbf{k} \cdot \mathbf{a}_{3}} \\
t_{2} e^{i \mathbf{k} \cdot \mathbf{a}_{3}} & t_{1} & t_{3} e^{i \mathbf{k} \cdot \mathbf{a}_{2}} \\
t_{1} e^{i \mathbf{k} \cdot \mathbf{a}_{2}} & t_{3} e^{i \mathbf{k} \cdot \mathbf{a}_{3}} & t_{2}
\end{array}\right), \\
H_{B A} & =H_{A B}^{\dagger}, \\
G_{A A} & =G_{B B}=0, \\
G_{A B} & =\Delta\left(\begin{array}{ccc}
1 & e^{i \mathbf{k} \cdot \mathbf{a}_{2}} & e^{i \mathbf{k} \cdot \mathbf{a}_{3}} \\
e^{i \mathbf{k} \cdot \mathbf{a}_{3}} & 1 & e^{i \mathbf{k} \cdot \mathbf{a}_{2}} \\
e^{i \mathbf{k} \cdot \mathbf{a}_{2}} & e^{i \mathbf{k} \cdot \mathbf{a}_{3}} & 1
\end{array}\right), \\
G_{B A} & =G_{A B}^{\dagger} .
\end{aligned}
$$

Here $\rho_{\uparrow}$ and $\rho_{\downarrow}$ are the order parameters for density waves in the up and down spin, respectively, $S_{\mathbf{k} \uparrow / \downarrow}=i \lambda_{\uparrow / \downarrow}\left(e^{i \mathbf{k} \cdot \mathbf{a}_{1}}+\right.$ $\left.e^{i \mathbf{k} \cdot \mathbf{a}_{2}}+e^{i \mathbf{k} \cdot \mathbf{a}_{3}}\right)$ are the Fourier transform of the second-nearestneighbor hopping with $\lambda_{\uparrow}$ and $\lambda_{\downarrow}$ being the order parameter for topological order in the up and down spin, and the vectors $\mathbf{a}_{1}, \mathbf{a}_{2}$, and $\mathbf{a}_{3}$ are (hexagonal) lattice vectors. [ $\mathbf{a}_{1}=a \hat{x}, \mathbf{a}_{2 / 3}=$ $\left.a\left(\frac{1}{2} \hat{x} \pm \frac{\sqrt{3}}{2} \hat{y}\right)\right]$. Following Weeks and Franz ${ }^{20}$ we parametrize the lattice distortion by $t_{j}=t+\delta t+\eta_{j}$ where $\delta t$ is a uniform shift and the Kekule texture is given by $\eta_{j}=\eta \cos \left(\frac{2 \pi}{3} j+\phi\right)$ with $\eta$ being the order parameter and $\phi$ a free parameter, which does not affect the size of the gap. The down-spin part of the generating Hamiltonian $\hat{h}_{\downarrow}$ is obtained from $\hat{h}_{\uparrow}$ by replacing $\rho_{\uparrow} \rightarrow \rho_{\downarrow}$ and $\lambda_{\uparrow} \rightarrow \lambda_{\downarrow}$.

The trial wave function, which is the ground state of the Hamiltonian in Eq. (5), is used to calculate the full interacting variational energy. This is done by calculating the expectation of the Hamiltonian:

$$
\mathcal{H}=-t \sum_{\langle i j\rangle} \sum_{\sigma} c_{i \sigma}^{\dagger} c_{j \sigma}+U \sum_{i} n_{i \uparrow} n_{i \downarrow}+\sum_{i \delta} V_{\delta} n_{i} n_{i+\delta},
$$

where $U$ represents an effective on-site interaction and $V_{\delta}$ represents the interaction between two sites separated by the distance $\delta$, which is taken up to the seventh neighbor and 


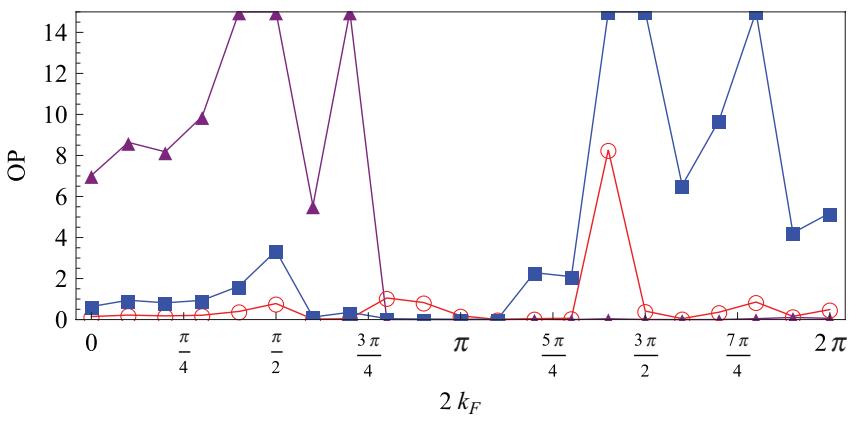

FIG. 2. (Color online) An example of order-parameter magnitudes as a function of the metal's Fermi wavelength (measured in units of $1 / a$ where $a=2.4 \AA$ is the graphene lattice constant). The data are given for a constant $U=0$ and $V_{0}=4 t$. The curves represent the order-parameter magnitude in units of the bare hopping $t$ with square (blue) charge-density wave, circle (red) anomalous Hall, and triangle (purple) superconductivity.

is given by the coupling $\alpha^{2}$ times the RKKY interaction in Eq. (4). The on-site interaction is estimated at $3.5 \mathrm{eV}$ (about $1.3 t),{ }^{35}$ but since the uncertainty is large, we choose to scan different values of $U$. The grid size we use is $64 \times 64$ unit cells (of six atoms each). In order to save computing time we calculate the correlation functions in momentum space, Fourier transform them, and use the resulting real-space correlations to evaluate the interaction energy for any given distance. The next step is to minimize the energy with respect to the order parameters and the minimum determines the mean-field ground state. We use the standard downhill simplex method $^{36}$ to do this minimization. The results are shown in Figs. 1 and 2.

\section{SUMMARY AND DISCUSSION}

In this paper we analyzed the effect of a metallic environment on fermions residing in a honeycomb lattice. The main idea is that the environment, through its Lindhard response, can induce long-range interactions in the honeycomb lattice, which are conducive to the formation of topological phases, such as the anomalous and spin Hall states. Our analysis consisted of a variational study of the many possible orders of a honeycomb lattice in the presence of an oscillating long-range interaction. To test our analysis, and to make a connection with previously derived results, we used an interaction cutoff at the next-nearest-neighbor length and plot a phase diagram for a spinless $V_{1}-V_{2}$ model. Our results are similar to those obtained by Weeks and Franz ${ }^{20}$ when spinless Fermions are considered. It is interesting to note that the Kekule phase vanishes as soon as the spin and on-site interaction are introduced.

When we introduce the oscillating long-range component of the interaction, we indeed find that topological phases, among other phases, may be induced in a honeycomb lattice through interaction with a metallic environment. In particular, topological order occurs in the case of strong coupling between the two species, when the Fermi wave number of the metal is between $\pi / 4 a$ and $\pi / 2 a$ (where $a$ is the lattice constant of the honeycomb). This is shown in Fig. 1, where the anomalous Hall and spin Hall effects are marked by open symbols.
The most promising path for realizing our proposal is in a cold-atoms context. A cold-atoms realization would consist of using two species of fermionic gases. The first would be confined to a honeycomb lattice (and could have one or two hyperfine states to imitate spinless or spin half particles). The second species would be confined to the honeycomb lattice plane, but without being sensitive to the optical lattice that traps the first species. This setup allows the realization of contact interactions between the two species through a Feshbach resonance, as well as the on-site intraspecies interactions described in Eq. (1) without significant limitations.

A realization of the scheme using electrons would employ a gated graphene layer. Unfortunately, it suffers from significant drawbacks, since it is a long-range Coulomb interaction that couples between the graphene and the gate. First, when $U=0, t$ [see Figs. 1(a) and 1(b)], the coupling constant $\alpha^{2}$ that is needed in order to induce topological order is about $2 t^{2}$. Defining $d$ as the distance between the layers, the interaction parameter is $\alpha=\frac{e^{2}}{d} \frac{\pi}{k_{F} a} \exp \left(-2 k_{F} d\right)$. The exponential suppression is the result of convolving the density modulations in the gate with the Coulomb potential as discussed in the Appendix. Even choosing a gate as close as $d=6 \AA$ yields $\alpha \sim t / 50$.

In addition, the large wave vector needed in the gate corresponds to a large density of the two-dimensional electron gas, which is very difficult to achieve in metals or semiconductors. Perhaps this could be mitigated by using doped graphene as the metallic layer to induce the interactions. At low density, the Friedel oscillations and resulting RKKY interactions in graphene are the result of scattering in the vicinity of the same valley point, i.e., short wave vector, and between different valleys. While intravalley scattering is suppressed due to the Klein paradox and decay as $1 / r^{3}$, the intervalley scattering is allowed and is of the right order of magnitude for our purposes. ${ }^{37,38}$ This suggests that the ideal gate for the realization of topological phases (if sufficient proximity could be achieved) is another layer of graphene that rotates by $30^{\circ}$ with respect to the bottom one and is doped such that the intervalley scattering vector matches the ideal wave vector. The challenge then becomes to prevent the tunneling between the two rather close graphene planes. Note that a similar setup was considered in Refs. 39-41.

\section{ACKNOWLEDGMENTS}

The authors would like to acknowledge useful discussions with J. Alicea, M. Franz, J. Lau, N. H. Lindner, and J. Simon. G.R. is grateful for the generous support of the Packard Foundation and the FENA Focus Center, one of six research centers funded under the Focus Center Research Program (FCRP), a Semiconductor Research Corporation entity. T.P.B. and G.R are supported by the Research Corporation Cottrell Scholars Award, and DARPA. T.P.B. was also supported by the National Science and Engineering Council of Canada.

\section{APPENDIX: ESTIMATE OF THE INDUCED INTERACTION IN THE GRAPHENE LAYER}

In this section we concentrate on the electronic realization of our proposal, and consider the details of gate-induced 
interaction between two points on a graphene sheet, $r_{1}^{\prime}$ and $r_{2}^{\prime}$, whose distance is $R$, taking into account screening, and the distance $d$ to the gate. We use the known result for the interaction between these points and two points on the metallic layer $r_{1}$ and $r_{2}$, which are close to $r_{1}^{\prime}$ and $r_{2}^{\prime}$ but not necessarily the same. The interaction term is therefore

$$
e^{4} \int d^{2} r_{1}^{\prime} d^{2} r_{2}^{\prime} \int d^{2} r_{1} d^{2} r_{2} \frac{n^{g}\left(r_{1}^{\prime}\right) n^{m}\left(r_{1}\right) n^{g}\left(r_{2}^{\prime}\right) n^{m}\left(r_{2}\right)}{\left|r_{1}-r_{2}^{\prime}\right|\left|r_{2}-r_{2}^{\prime}\right|} .
$$

We would like to integrate out the metal by integrating over the positions $r_{i}$ :

$$
\approx e^{4} \int d^{2} r_{1}^{\prime} d^{2} r_{2}^{\prime} n^{g}\left(r_{1}^{\prime}\right) n^{g}\left(r_{2}^{\prime}\right) \int d^{2} r_{1} d^{2} r_{2} \frac{\left\langle n^{m}\left(r_{1}\right) n^{m}\left(r_{2}\right)\right\rangle}{\left|r_{1}-r_{2}^{\prime}\right|\left|r_{2}-r_{2}^{\prime}\right|}=\int d^{2} r_{1}^{\prime} d^{2} r_{2}^{\prime} n^{g}\left(r_{1}^{\prime}\right) n^{g}\left(r_{2}^{\prime}\right) V_{e f f}\left(r_{1}^{\prime}, r_{2}^{\prime}\right) .
$$

The effective interaction can be calculated using the polarization of the metal:

$$
\begin{aligned}
V_{e f f} & =e^{4} \frac{1}{a^{2}} \int d^{2} r_{1} d^{2} r_{2} \frac{\left\langle n^{m}\left(r_{1}\right) n^{m}\left(r_{2}\right)\right\rangle}{\left|r_{1}-r_{1}^{\prime}\right|\left|r_{2}-r_{2}^{\prime}\right|} \\
& =-e^{4} D(0) \frac{1}{a^{2}} \int d^{2} \rho_{1} d^{2} \rho_{2} \frac{\cos \left[2 k_{F}\left(\rho_{1}-\rho_{2}\right)\right]}{\left[2 k_{F}\left(\rho_{1}-\rho_{2}\right)\right]^{2}} \frac{1}{\sqrt{d^{2}+\left(\rho_{1}-\rho_{1}^{\prime}\right)^{2}} \sqrt{d^{2}+\left(\rho_{2}-\rho_{2}^{\prime}\right)^{2}}},
\end{aligned}
$$

where $D(0)=1 / 2 t a^{2}$ is the metallic density of states at the Fermi level and $a \approx 2.4 \AA$ is the lattice constant. We have explicitly written the distances $\left|r_{1}-r_{1}^{\prime}\right|$ and $\left|r_{2}-r_{2}^{\prime}\right|$ taking into account the distance between the layers $d$ and denoting the distance in each layer by $\rho$. We rewrite the effective interaction

$$
V_{e f f}=-\frac{e^{4}}{2 t a^{4}} \int \rho_{1} d \rho_{1} d \theta_{1} \int \rho_{2} d \rho_{2} d \theta_{2} \frac{\cos \left(2 k_{F} x\right)}{\left(2 k_{F} x\right)^{2} \sqrt{\left(d^{2}+\rho_{1}^{2}\right)\left(d^{2}+\rho_{2}^{2}\right)}},
$$

where we measure $\rho_{1}$ from $\rho_{1}^{\prime}$ and $\rho_{2}$ from $\rho_{2}^{\prime}$ in the plane. With this choice $x=$ $\sqrt{R^{2}+\rho_{1}^{2}+\rho_{2}^{2}-2 R\left[\rho_{1} \cos \left(\theta_{1}\right)-\rho_{2} \cos \left(\theta_{2}\right)\right]-2 \rho_{1} \rho_{2} \cos \left(\theta_{1}-\theta_{2}\right)}$ is the distance between $\rho_{1}$ and $\rho_{2}$. Now, we assume that $R=\left|\rho_{1}^{\prime}-\rho_{2}^{\prime}\right|$ is larger than $\rho_{1}, \rho_{2}$ in the relevant part of the integral (basically assuming $|R| \ll d$ ) and we can simplify the expression. We also replace $x^{2}$ in the denominator by $R^{2}$ and linearize in $r / R$ in the cosine. This gives

$$
\begin{aligned}
V_{e f f} & \approx-\frac{e^{4}}{2 t a^{4}\left(2 k_{F} R\right)^{2}} \int \rho_{1} d \rho_{1} d \theta_{1} \int \rho_{2} d \rho_{2} d \theta_{2} \frac{\cos \left\{2 k_{F}\left[R-\rho_{1} \cos \left(\theta_{1}\right)+\rho_{2} \cos \left(\theta_{2}\right)\right]\right\}}{\sqrt{\left(d^{2}+\rho_{1}^{2}\right)\left(d^{2}+\rho_{2}^{2}\right)}} \\
& =-2 \pi \frac{e^{4}}{2 t a^{4}\left(2 k_{F} R\right)^{2}} \int \rho_{1} d \rho_{1} \int \rho_{2} d \rho_{2} d \theta_{2} \frac{J_{0}\left(2 k_{F} \rho_{1}\right) \cos \left\{2 k_{F}\left[R+\rho_{2} \cos \left(\theta_{2}\right)\right]\right\}}{\sqrt{\left(d^{2}+\rho_{1}^{2}\right)\left(d^{2}+\rho_{2}^{2}\right)}} \\
& =-(2 \pi)^{2} \frac{e^{4}}{2 t a^{4}\left(2 k_{F} R\right)^{2}} \int \rho_{1} d \rho_{1} \int \rho_{2} d \rho_{2} \frac{J_{0}\left(2 k_{F} \rho_{1}\right) J_{0}\left(2 k_{F} \rho_{2}\right) \cos \left(2 k_{F} R\right)}{\sqrt{\left(d^{2}+\rho_{1}^{2}\right)\left(d^{2}+\rho_{2}^{2}\right)}} \\
& =-(2 \pi)^{2} \frac{e^{4} \cos \left(2 k_{F} R\right)}{2 t a^{4}\left(2 k_{F} R\right)^{2}}\left(\int \rho d \rho \frac{J_{0}\left(2 k_{F} \rho\right)}{\sqrt{\left(d^{2}+\rho^{2}\right)}}\right)^{2} \\
& =-(2 \pi)^{2} \frac{e^{4} \cos \left(2 k_{F} R\right)}{2 t a^{4}\left(2 k_{F} R\right)^{2}}\left(\frac{e^{-2 k_{F} d}}{2 k_{F}}\right)^{2} \\
& =-\pi^{2} \frac{e^{4} \frac{\cos \left(2 k_{F} R\right)}{8 t a^{4} k_{F}^{4} R^{2}} e^{-4 k_{F} d},}{}
\end{aligned}
$$

where we have used $\int d \theta \cos [a+b \cos (\theta)]=2 \pi J_{0}(b) \cos (a)$ in the angular integrals. Indeed, this expression is suppressed by $\frac{(2 \pi)^{2}}{\left(2 k_{F} a\right)^{2}} \exp \left(-4 k_{F} d\right)$ with respect to the bare coupling square.

${ }^{1}$ C. L. Kane and E. J. Mele, Phys. Rev. Lett. 95, 146802 (2005).

${ }^{2}$ C. L. Kane and E. J. Mele, Phys. Rev. Lett. 95, 226801 (2005).

${ }^{3}$ F. D. M. Haldane, Phys. Rev. Lett. 61, 2015 (1988).

${ }^{4}$ H. Min, J. E. Hill, N. A. Sinitsyn, B. R. Sahu, L. Kleinman, and A. H. MacDonald, Phys. Rev. B 74, 165310 (2006).
${ }^{5}$ H. Min, J. E. Hill, N. A. Sinitsyn, B. R. Sahu, L. Kleinman, and A. H. MacDonald, Phys. Rev. B 74, 165310 (2006).

${ }^{6}$ H.-M. Guo and M. Franz, Phys. Rev. B 80, 113102 (2009).

${ }^{7}$ H.-M. Guo and M. Franz, Phys. Rev. Lett. 103, 206805 (2009).

${ }^{8}$ A. Rüegg, J. Wen, and G. A. Fiete, Phys. Rev. B 81, 205115 (2010). 
${ }^{9}$ H. Zhang, C.-X. Liu, X.-L. Qi, X. Dai, Z. Fang, and S.-C. Zhang, Nat. Phys. 5, 438 (2009).

${ }^{10}$ D. Hsieh, D. Qian, L. Wray, Y. Xia, Y. S. Hor, R. J. Cava, and M. Z. Hasan, Nature (London) 452, 970 (2008).

${ }^{11}$ Y. Xia, D. Qian, D. Hsieh, L. Wray, A. Pal, H. Lin, A. Bansil, D. Grauer, Y. S. Hor, R. J. Cava et al., Nat. Phys. 5, 398 (2009).

${ }^{12}$ M. König, S. Wiedmann, C. Brüne, A. Roth, H. Buhmann, L. W. Molenkamp, X.-L. Qi, and S.-C. Zhang, Science 318, 766 (2007).

${ }^{13}$ A. Roth, C. Brüne, H. Buhmann, L. W. Molenkamp, J. Maciejko, X.-L. Qi, and S.-C. Zhang, Science 325, 294 (2009).

${ }^{14}$ L. Tarruell, D. Greif, T. Uehlinger, G. Jotzu, and T. Esslinger, e-print arXiv:1111.5020 (to be published).

${ }^{15}$ T. D. Stanescu, V. Galitski, and S. Das Sarma, Phys. Rev. A 82, 013608 (2010).

${ }^{16}$ P. R. Wallace, Phys. Rev. 71, 622 (1947).

${ }^{17}$ S. Zhou, G.-H. Gweon, A. Fedorov, P. First, W. de Heer, D.-H. Lee, F. Guinea, A. Castro Neto, and A. Lanzara, Nat. Mater. 6, 770 (2007).

${ }^{18}$ O. Bahat-Treidel, O. Peleg, M. Grobman, N. Shapira, M. Segev, and T. Pereg-Barnea, Phys. Rev. Lett. 104, 063901 (2010).

${ }^{19}$ S. Raghu, X.-L. Qi, C. Honerkamp, and S.-C. Zhang, Phys. Rev. Lett. 100, 156401 (2008).

${ }^{20}$ C. Weeks and M. Franz, Phys. Rev. B 81, 085105 (2010).

${ }^{21}$ J. Wen, A. Rëgg, C.-C. J. Wang, and G. A. Fiete, Phys. Rev. B 82, 075125 (2010).

${ }^{22}$ I. F. Herbut, Phys. Rev. Lett. 97, 146401 (2006).

${ }^{23}$ I. F. Herbut, V. Juričić, and B. Roy, Phys. Rev. B 79, 085116 (2009).
${ }^{24}$ I. F. Herbut, V. Juričić, and O. Vafek, Phys. Rev. B 80, 075432 (2009).

${ }^{25}$ M. Ruderman and C. Kittel, Phys. Rev. 96, 99 (1954).

${ }^{26}$ T. Kasuya, Prog. Theor. Phys. 16, 45 (1956).

${ }^{27}$ K. Yosida, Phys. Rev. 106, 893 (1957).

${ }^{28}$ P. Simon, B. Braunecker, and D. Loss, Phys. Rev. B 77, 045108 (2008).

${ }^{29} \mathrm{G}$. Giuliani and G. Vignale, Quantum Theory of the Electron Liquid (Cambridge University Press, Cambridge, England, 2005).

${ }^{30}$ P. M. Chaikin and T. C. Lubensky, Principles of Condensed Matter Physics (Cambridge University Press, Cambridge, England, 2000).

${ }^{31}$ C. Chamon, Phys. Rev. B 62, 2806 (2000).

${ }^{32}$ C.-Y. Hou, C. Chamon, and C. Mudry, Phys. Rev. Lett. 98, 186809 (2007).

${ }^{33}$ B. Uchoa and A. H. Castro Neto, Phys. Rev. Lett. 98, 146801 (2007).

${ }^{34}$ B. Roy and I. F. Herbut, Phys. Rev. B 82, 035429 (2010).

${ }^{35}$ O. V. Yazyev, Phys. Rev. Lett. 101, 037203 (2008).

${ }^{36}$ W. H. Press, S. A. Teukolsky, W. T. Vetterling, and B. P. Flannery, Numerical Recipes Electronic Edition (Cambridge University Press, Cambridge, England, 1997).

${ }^{37}$ C. Bena, Phys. Rev. Lett. 100, 076601 (2008).

${ }^{38}$ T. Pereg-Barnea and A. H. MacDonald, Phys. Rev. B 78, 014201 (2008).

${ }^{39}$ H. Min, R. Bistritzer, J.-J. Su, and A. H. MacDonald, Phys. Rev. B 78, 121401 (2008).

${ }^{40}$ B. Seradjeh, H. Weber, and M. Franz, Phys. Rev. Lett. 101, 246404 (2008).

${ }^{41}$ C.-H. Zhang and Y. N. Joglekar, Phys. Rev. B 77, 233405 (2008). 